\title{
Review Article \\ FCM Clustering Algorithms for Segmentation of Brain MR Images
}

\author{
Yogita K. Dubey and Milind M. Mushrif \\ Department of Electronics and Telecommunication, Yeshwantrao Chavan College of Engineering, Wanadongri, \\ Hingna Road, Nagpur, Maharashtra 441110, India
}

Correspondence should be addressed to Yogita K. Dubey; yogeetakdubey@yahoo.co.in

Received 8 November 2015; Revised 16 February 2016; Accepted 17 February 2016

Academic Editor: Rustom M. Mamlook

Copyright ( 2016 Y. K. Dubey and M. M. Mushrif. This is an open access article distributed under the Creative Commons Attribution License, which permits unrestricted use, distribution, and reproduction in any medium, provided the original work is properly cited.

\begin{abstract}
The study of brain disorders requires accurate tissue segmentation of magnetic resonance (MR) brain images which is very important for detecting tumors, edema, and necrotic tissues. Segmentation of brain images, especially into three main tissue types: Cerebrospinal Fluid (CSF), Gray Matter (GM), and White Matter (WM), has important role in computer aided neurosurgery and diagnosis. Brain images mostly contain noise, intensity inhomogeneity, and weak boundaries. Therefore, accurate segmentation of brain images is still a challenging area of research. This paper presents a review of fuzzy c-means (FCM) clustering algorithms for the segmentation of brain MR images. The review covers the detailed analysis of FCM based algorithms with intensity inhomogeneity correction and noise robustness. Different methods for the modification of standard fuzzy objective function with updating of membership and cluster centroid are also discussed.
\end{abstract}

\section{Introduction}

The purpose of image segmentation is to partition image to different regions, based on given criteria for future processing. Image segmentation plays an important role in medical applications such as abnormality detection, quantitative analysis, and postsurgical assessment. Due to unknown noise, intensity inhomogeneity, and partial volume effect, their precise segmentation is a difficult task. A variety of fuzzy techniques [1-3] have been reported in the literature for image segmentation. These methods fail to deal with local spatial property of images which leads to strong noise sensibility. Brain MR images mainly suffer from intensity inhomogeneity and noise caused due to radio frequency coil used in image acquisition $[4,5]$. Therefore correction of intensity inhomogeneity as well as removal of noise is always desirable before segmentation of brain MR images.

Segmentation of MR images is still a challenging problem because they are affected by multiple factors such as

(1) noise caused in image acquisition,

(2) poor contrast and intensity inhomogeneity physically linked to the radio frequency MR signal,
(3) partial volume effect being the mixture of several tissue signals in the same pixel, induced by the image resolution.

Few review papers for the segmentation of brain MR images with intensity inhomogeneity correction and noise robustness have been published. In [6], the performances of four methods (a phantom correction method, an image smoothing technique, homomorphic filtering, and surface fitting approach) have been investigated. In [7], quantitative and qualitative evaluation of six retrospective methods (low pass filtering, homomorphic filtering, Fourier domain, nonparametric, histogram based, and model based methods) have been reported. In [8], review of intensity correction has been categorized in model based methods such as low frequency, hyper surface, and statistical models. Reviews discussed in [9-11] are mainly based on intensity inhomogeneity correction methods that are categorized as prospective and retrospective methods only.

In [9], retrospective methods are divided into two categories such as gray scale based and transformed domain based. Review discussed different surface fitting (polynomial fitting, spline fitting), spatial filtering (low pass filtering and 
homomorphic filtering), and statistical methods (Markov random fields (MRF) and FCM) under the category of gray scale based methods and probability density function, Fourier domain, and wavelet domain methods under the category of transform based methods.

Prospective methods like phantom, multicoil, and special sequences and retrospective methods such as filtering methods (homomorphic and unsharp masking), surface fitting (intensity and gradient), segmentation based (maximum likelihood (ML), maximum a posteriori (MAP), FCM, and nonparametric methods), and histogram based (high frequency maximization, information minimization, and histogram matching) methods are discussed in [10]. This review also analysed numerous publications to assess major trends, popularity, and applications of intensity inhomogeneity correction methods.

Different brain image denoising methods such as anisotropic nonlinear diffusion, MRF, wavelet based, analytical correction, and nonlocal methods are discussed in [11]. This review also discussed prospective methods like phantom, multicoil, and special sequences and retrospective methods such as surface fitting methods, segmentation based (ML, MAP, and FCM), histogram based, and filtering methods. Main focus of this paper is as follows:

(1) Comprehensive review of FCM clustering based algorithms for the segmentation of brain MR images with intensity inhomogeneity corrections and noise robustness is presented.

(2) Complete mathematical analysis for the formulation of objective function of FCM clustering based algorithms for intensity inhomogeneity correction and noise robustness is carried out.

(3) Algorithms are compared on the basis of updating of membership function and cluster centroid.

(4) Computational complexity and noise robustness of these algorithms are discussed.

(5) Quantitative measures used by researcher are also discussed.

Rest of the paper is organized as follows: Intensity inhomogeneity in brain MR images and its correction methods are described in Section 2. Noise in brain MR images and denoising methods are briefed in Section 3. FCM clustering algorithm and its drawback for the segmentation of brain MR image are briefly explained in Section 4. Detailed analysis of FCM clustering based algorithms for the segmentation of brain MR images with intensity inhomogeneity correction and noise robustness is presented in Sections 5, 6, and 7. Section 8 describes the different validation methods which are used for comparison. Information about the dataset is given in Section 9, followed by the conclusion of the study in Section 10.

\section{Intensity Inhomogeneity in Brain MR Images and Its Correction Methods}

In brain MR images, the observed signal is modelled as a product of the true signal and spatial varying factor called bias field:

$$
Y_{k}=X_{k} G_{k}, \quad 1 \leq k \leq N,
$$

where $X_{k}$ and $Y_{k}$ are the true and observed intensities at the $k$ th pixel, respectively, $G_{k}$ is the bias field, and $N$ is the total number of pixels in a brain MR image. Intensity inhomogeneity or bias is the slowly changing and smooth variation in signal intensity. For a bias field of magnitude $40 \%$, the signal is multiplied by a field with values ranging from 0.8 to 1.2 (i.e., with values between $20 \%$ below true intensity and $20 \%$ above true intensity). The application of logarithmic transformation to the intensities allows artifact to be modelled as additive bias field [12] as given below:

$$
y_{k}=x_{k}+\beta_{k}, \quad 1 \leq k \leq N \text {. }
$$

Figure 1 depicts the effect of intensity inhomogeneity on brain MR images. Original brain MR image [13] is shown in Figure 1 (a) and images altered by $40 \%, 80 \%$, and $100 \%$ intensity inhomogeneity, respectively, are shown in Figures 1(b), 1(c), and 1(d), respectively.

Ahmed et al. [14] used sinusoidal gain field of higher spatial frequency as bias field. In [15-17], the bias field is estimated by a linear combination of a set of basis functions as

$$
b_{k}=\sum_{1}^{M} \bar{\omega}_{k} g_{k}(k)=\bar{\omega}^{T} G(K),
$$

where $\bar{\omega}^{T}=\left(\bar{\omega}_{1}, \bar{\omega}_{2}, \ldots, \bar{\omega}_{M}\right)^{T}, \bar{\omega}_{k} \in \Re, k=1,2, \ldots, M$, are the combination coefficients. $G(k)=\left(g_{1}(k), g_{2}(k), \ldots, g_{M}(k)\right)^{T}$ are the set of basis functions. In $[18,19]$, weighted bias field is used with initialization of weights as $\omega_{k} \in(0,1)$, for example, with 10 data items $\omega_{1}=0.008$, increase $0.001, \omega_{2}, \omega_{3}, \ldots, \omega_{10}$.

2.1. FCM Clustering Methods with Spatial Constraints. The FCM based methods are modified by incorporating spatial constraint in the objective function of FCM. These methods $[12,20]$ are used for intensity inhomogeneity correction and partial volume segmentation of brain MR images. Ahmed et al. [14] proposed bias corrected FCM (BCFCM) by modifying the objective function of FCM to compensate for the intensity inhomogeneity. But BCFCM is very timeconsuming, since it computes the neighbourhood term in each iteration step. This drawback is eliminated in spatially constrained kernelized FCM (SKFCM) [21], where different penalty terms containing spatial neighbourhood information in the objective function are used. They also replaced the similarity measurement in the FCM by a kernel induced distance.

2.2. Modified FCM Based Methods. Improved and enhanced FCM clustering algorithms [22-27] have been used to 


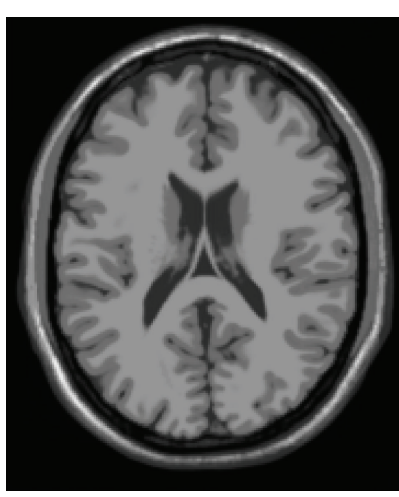

(a)

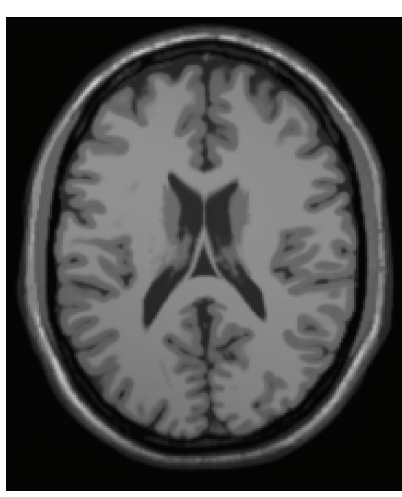

(b)

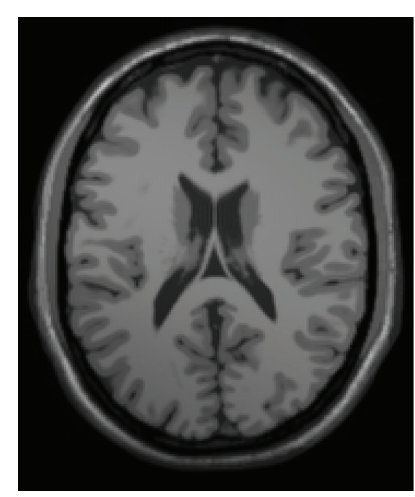

(c)

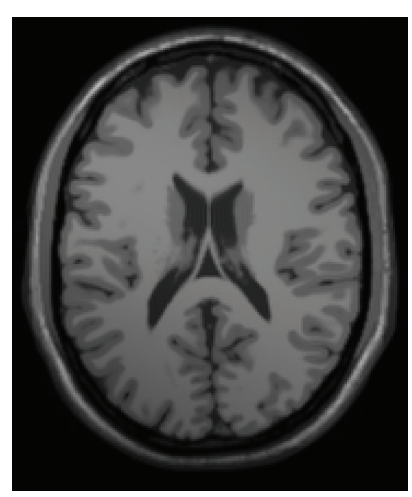

(d)

FIGURE 1: Intensity inhomogeneity in brain MR images. (a) Original brain MR image. The images altered by $40 \%, 80 \%$, and $100 \%$ intensity inhomogeneity in (b), (c), and (d), respectively.

accelerate the image segmentation process and to correct the intensity inhomogeneity during segmentation. Integration of fuzzy spatial relations in deformable models is proposed in $[28,29]$ for brain MRI segmentation.

2.3. Other Methods. In recent years, multilevel methods [30, 31], multidimensional approach [32], multichannel [33, 34], model based approaches [35-38], and level set approaches [39-42] have been widely used for brain image segmentation and tumor detection.

2.4. Generalized FCM Based Methods. Modified fast FCM algorithms $[15,43,44]$, rough set based FCM clustering algorithms $[17,45,46]$, possibilistic FCM clustering algorithm [16], and FCM based algorithms [18, 19, 47-49] are also used for brain image segmentation. Recently intuitionistic fuzzy set based clustering $[50,51]$ approaches have been used for brain image segmentation.

\section{Noise in Brain MR Images and Denoising Methods}

Image segmentation algorithms are sensitive to noise. The presence of noise produces undesirable visual quality and lowers the visibility of low contrast objects. Effect of noise on brain MR images is shown in Figure 2. Original brain MR image [13] is shown in Figure 2(a) and images corrupted by $3 \%, 7 \%$, and $11 \%$ noise are shown in Figures 2(b), 2(c), and 2(d), respectively. Thus image denoising is one of the important steps as a preprocessing in various applications. Its objective is to recover the best estimate of the original image from its noisy version. The performance of segmentation algorithms can be improved by image filtering. Linear low pass filters update value of a pixel by average of its neighbourhoods. These filters reduce noise but produce blurred images and fail to preserve the edges in the presence of large amount of noise. Nonlinear filters have better performance in edge preserving but degrade fine structure; therefore, the resolution of the image is reduced. Wavelet filtering can overcome this drawback. Wavelet transform at high frequency gives good spatial resolution, while at low frequency it gives good frequency resolution. Thus different image denoising methods have advantages and disadvantages in terms of computation cost, quality of denoising, and boundary preserving. Therefore, denoising is still an open issue and needs an improvement.

3.1. Nonlocal Filters. Coupe et al. [52] proposed optimized block-wise nonlocal means denoising filter for 3D magnetic resonance images which uses the natural redundancy of information in image to remove the noise. Liu et al. [53] proposed enhanced nonlocal means denoising filter for 3D magnetic resonance images. The filter was designed considering characteristics of Rician noise in the MR images. Manjón et al. [54] proposed adaptive nonlocal means denoising method for MR images with spatially varying noise levels. Hu et al. [55] presented improved DCT-based nonlocal means filter for MR image denoising.

3.2. Anisotropic Diffusion Filters. The anisotropic diffusion filtering is widely used for MR image enhancement. It is a general scale-space approach to edge detection, introduced in [56]. Fully automatic method for the segmentation of MR brain images is proposed in [57]. The method used probabilistic anisotropic diffusion to suppress the influence of extracerebral tissue in brain MR images and multiscale watersheds for image segmentation. Samsonov and Johnson [58] proposed noise-adaptive nonlinear diffusion filtering of MR images with spatially varying noise levels. They use a priori information regarding the image noise level spatial distribution for the local adjustment of the anisotropic diffusion filter. Krissian and Aja-Fernandez [59] presented noise-driven anisotropic diffusion filtering to remove Rician noise from magnetic resonance images. This filter relies on a robust estimation of the standard deviation of the noise.

3.3. Wavelet Based Methods. Wavelet based methods are proposed by Nowak [60], Alexander et al. [61], Bao and Zhang [62], and Anand and Sahambi [63] for noise suppression in magnetic resonance images. Xue et al. [64] presented 


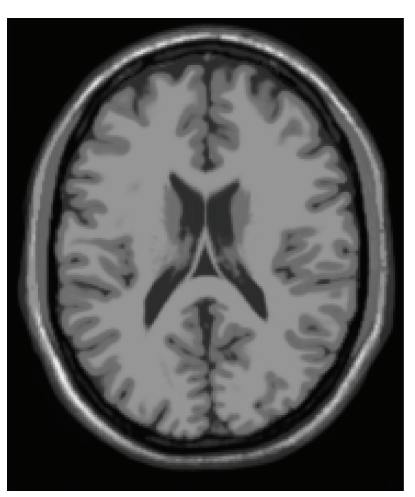

(a)

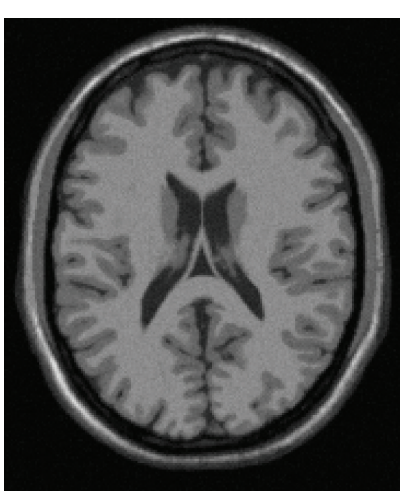

(b)

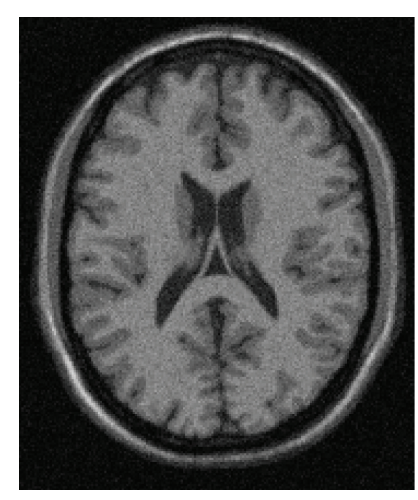

(c)

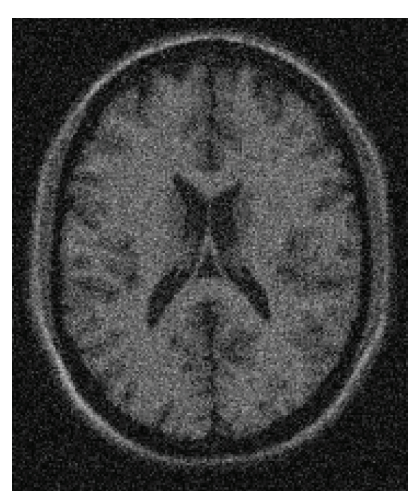

(d)

FIGURE 2: Noise in brain MR images. (a) Original brain MR image. The images corrupted by 3\%, 7\%, and 11\% noise in (b), (c), and (d), respectively.

an integrated method of the adaptive enhancement for an unsupervised global-to-local segmentation of brain tissues in three-dimensional (3D) MR images where wavelet filter is used to denoise the image and segmentation is carried out by combining spatial feature based fuzzy clustering.

\section{FCM Clustering}

FCM is the most effective algorithm for data clustering. FCM was proposed by Dunn [65] and later on it was modified by Bezdek [66]. The standard FCM objective function for partitioning the data $\left\{x_{k}\right\}_{k=1}^{N}$ into $c$ clusters is given as

$$
J_{\mathrm{FCM}}=(U, V)=\sum_{i=1}^{c} \sum_{k=1}^{N} \mu_{i k}^{p}\left\|x_{k}-v_{i}\right\|^{2},
$$

where $V=\left\{v_{i}\right\}_{i=1}^{c}$ are the prototype of cluster and array $U=$ $\left\{\mu_{i k}\right\}$ represents the partition matrix, $c$ is the number of cluster centroids, $N$ is the number of pixels or data points, $x_{k}$ is the $k$ th pixel, and $v_{i}$ is the centroid of $i$ th cluster. $\left\|x_{k}-v_{i}\right\|^{2}=$ $d_{i k}=d\left(x_{k}, v_{i}\right)$ is the distance measure between cluster center $v_{i}$ and the pixel $x_{k} \cdot \mu_{i k}$ is the fuzzy membership of $k$ th pixel $i$ th cluster. This membership value satisfies the conditions $\mu_{i k} \in$ $[0,1], 1 \leq i \leq c, 1 \leq k \leq N, 0<\sum_{k=1}^{N} \mu_{i k}<N, 1 \leq i \leq c$, and $\sum_{i=1}^{c} \mu_{i k}=1,1 \leq k \leq N$.

Parameter $p \in(1, \infty)$ is a weighing exponent on each membership ( 1 for hard clustering and increasing for fuzzy clustering). It determines the amount of fuzziness of the resulting classification and is usually set as 2 . The FCM objective function is minimized when high membership values are assigned to pixels which are close to the centroid of its particular class, and low membership values are assigned when pixels are away from the centroid [67]. The partition matrix and cluster centroid are updated as

$$
\begin{aligned}
\mu_{i k} & =\frac{1}{\sum_{j=1}^{c}\left(d_{i k}^{2} / d_{j k}^{2}\right)^{1 / p}}, \\
v_{i} & =\frac{\sum_{k=1}^{N} \mu_{i k}^{p} x_{k}}{\sum_{k=1}^{N} \mu_{i k}^{p}} .
\end{aligned}
$$

The drawback of FCM clustering for image segmentation is that its objective function does not take into consideration any spatial dependence among pixels of image but deals with images the same as separate points. Second drawback of FCM clustering method is that the membership function is mostly decided by $d\left(x_{k}, v_{i}\right)$, which measures the similarity between the pixel intensity and the cluster center. Higher membership depends on closer intensity values to the cluster center. Hence membership function is highly sensitive to noise. In MR image with noise and intensity inhomogeneity, this results in improper segmentation.

The result of FCM on brain MR images corrupted by noise and intensity inhomogeneity from McGill database [13] is shown in Figure 3. Figure 3(a) shows original image with $3 \%$ noise and $0 \%$ intensity inhomogeneity, Region of CSF, Region of GM, and Region of WM, respectively. Figure 3(b) shows original image with $0 \%$ noise and $40 \%$ intensity inhomogeneity, Region of CSF, Region of GM, and Region of WM, respectively. In case of noisy images, tissue class may differ and appears as salt and pepper noise. For example, few GM pixels are shown in the homogeneous region of WM as shown in Figure 3(a). In case of images, corrupted by intensity inhomogeneity, significant size of pixels in GM and WM may be erroneously classified as another class as shown in Figure 3(b). In order to deal with these issues, many variants of FCM are proposed. In these algorithms spatial information is incorporated in objective function of original FCM algorithm to improve the performance of image segmentation.

In the next sections, the comparative analysis of FCM based methods is presented. These methods are categorized as methods dealing with intensity inhomogeneity only, methods dealing with noise only, and methods dealing with intensity inhomogeneity and noise.

\section{FCM Based Methods with Intensity Inhomogeneity Correction}

This section gives the mathematical analysis of FCM based methods for the segmentation of brain MR images with intensity inhomogeneity correction. 

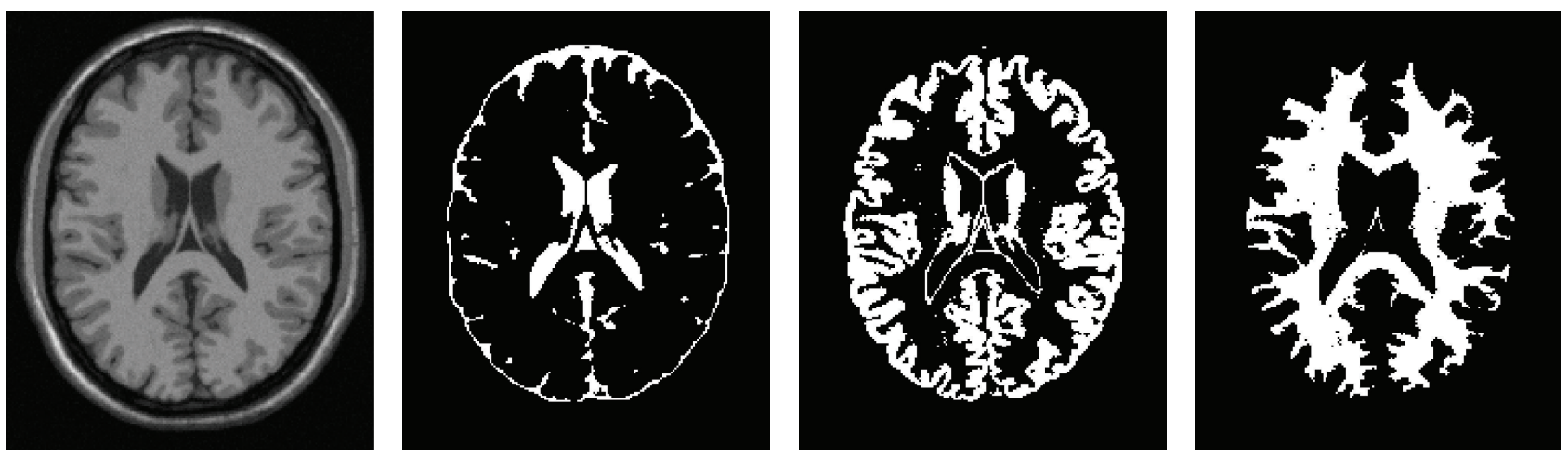

(a)
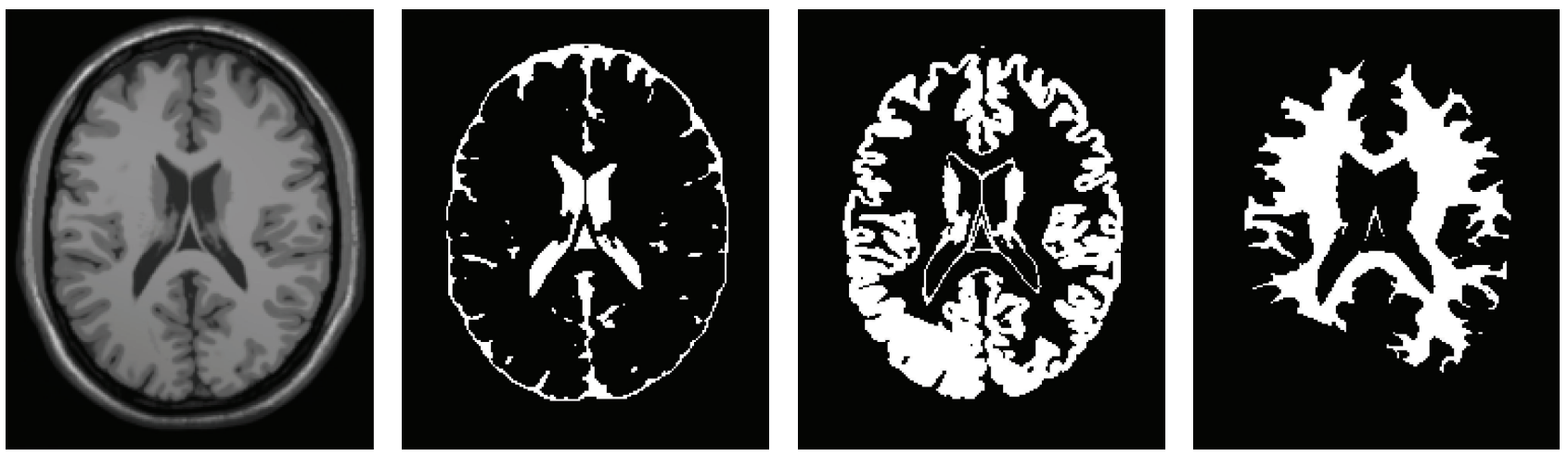

(b)

FIGURE 3: Result of FCM on brain MR images corrupted by noise and intensity variation. (a) Original image with $3 \%$ noise and $0 \%$ intensity inhomogeneity, Region of CSF, Region of GM, and Region of WM, respectively. (b) Original image with $0 \%$ noise and $40 \%$ intensity inhomogeneity, Region of CSF, Region of GM, and Region of WM, respectively.

5.1. Bias Corrected FCM (BCFCM). Ahmed et al. [14] proposed a modification in standard FCM objective function to deal with intensity inhomogeneity of brain MR images by introducing a term that allows the labelling of pixel to be influenced by the intensity of its immediate neighbourhood. Neighbourhood acts as a regularizer and biases the solution towards piece-wise homogeneous labelling and is useful in segmenting MR scan corrupted by salt and pepper noise. The modified objective function is given as

$$
\begin{aligned}
J= & \sum_{i=1}^{c} \sum_{k=1}^{N} \mu_{i k}^{p}\left\|y_{k}-\beta_{k}-v_{i}\right\|^{2} \\
& +\frac{\alpha}{N_{R}} \sum_{i=1}^{c} \sum_{k=1}^{N} \mu_{i k}^{p}\left(\sum_{y_{r} \in N_{k}}\left\|y_{r}-\beta_{r}-v_{i}\right\|^{2}\right),
\end{aligned}
$$

where $y_{k}$ is the observed log-transformed intensities at the $k$ th pixel and $N_{k}$ stands for set of neighbours that exists in a window around $x_{k}$ and is the cardinality of $N_{R}$. The effect of the neighbours term is controlled by parameter $\alpha$. The relative importance of the regularizing term is inversely proportional to signal-to-noise ratio (SNR) of the MRI signal. Lower SNR would require a higher value of the parameter $\alpha$.
The membership function, centroid, and bias field are updated as follows:

$$
\begin{aligned}
& \mu_{i k}^{*} \\
& =\frac{1}{\sum_{j=1}^{c}\left(\left(D_{i k}+\left(\alpha / N_{R}\right) \gamma_{i}\right) /\left(D_{j k}+\left(\alpha / N_{R}\right) \gamma_{j}\right)\right)^{1 /(p-1)}} \\
& v_{i}^{*}=\frac{\sum_{k=1}^{N} \mu_{i k}^{p}\left(\left(y_{k}-\beta_{k}\right)+\left(\alpha / N_{R}\right) \sum_{y_{r} \in N_{k}}\left(y_{r}-\beta_{k}\right)\right)}{(1+\alpha) \sum_{k=1}^{N} \mu_{i k}^{p}} \\
& \beta_{k}^{*}=y_{k}-\frac{\sum_{i=1}^{c} \mu_{i k}^{p} \nu_{i}}{\sum_{i=1}^{c} \mu_{i k}^{p}},
\end{aligned}
$$

where $D_{i k}=\left\|y_{k}-\beta_{k}-v_{i}\right\|^{2}$ and $\gamma_{i}=\sum_{y_{r} \in N_{k}} \| y_{r}-$ $\beta_{k}-v_{i} \|^{2}$. The BCFCM outperformed the FCM on both simulated and real MR images. In noisy images, the BCFCM technique produced better results and compensates for noise by including a regularization term.

5.2. FCM with Spatial Constraints. The BCFCM is computationally inefficient due to introduction of spatial constraints, insufficient robustness to outliers, and difficulty in clustering non-Euclidean structure data. Chen and Zhang [21] proposed 
a modified objective function which is computationally efficient as compared to BCFCM and is given as

$$
J=\sum_{i=1}^{c} \sum_{k=1}^{N} \mu_{i k}^{p}\left\|x_{k}-v_{i}\right\|^{2}+\alpha \sum_{i=1}^{c} \sum_{k=1}^{N} \mu_{i k}^{p}\left(\left\|\widetilde{x}_{k}-v_{i}\right\|^{2}\right),
$$

where $\tilde{x}_{k}$ is the sample mean of neighbouring pixels lying within a window around pixel $x(k)$ which can be computed in advance. The idea is that the term $\left(1 / N_{R}\right) \sum_{x_{r} \in N_{k}}\left\|x_{r}-v_{i}\right\|^{2}$ can be written as $\left(1 / N_{R}\right) \sum_{x_{r} \in N_{k}}\left\|x_{r}-\tilde{x}_{k}\right\|^{2}+\left\|\tilde{x}_{k}-v_{i}\right\|^{2}$. Thus the modified FCM algorithm with spatial constraints is iterated for minimizing objective function with the following update equations for membership function and centroid:

$$
\begin{aligned}
\mu_{i k} & =\frac{\left(\left\|x_{k}-v_{i}\right\|^{2}+\alpha\left\|\tilde{x}_{k}-v_{i}\right\|^{2}\right)^{-1 /(p-1)}}{\sum_{j=1}^{c}\left(\left\|x_{k}-v_{j}\right\|^{2}+\alpha\left\|\tilde{x}_{k}-v_{j}\right\|^{2}\right)^{-1 /(p-1)}}, \\
v_{i} & =\frac{\sum_{k=1}^{N} \mu_{i k}^{p}\left(x_{k}+\tilde{x}_{k}\right)}{(1+\alpha) \sum_{k=1}^{N} \mu_{i k}^{p}} .
\end{aligned}
$$

Above equations are computationally simpler than those obtained from [14]. Further, to enhance robustness of clustering, $\tilde{x}_{k}$ can be taken as median of neighbours with specified window around $x_{k}$. Chen and Zhang [21] named these algorithms with mean and median filtering as FCM_S1 and FCM_S2, respectively. In FCM_S1 and FCM_S2 the Euclidean distance $\left\|x_{k}-v_{i}\right\|^{2}$ was replaced with Gaussian kernel induced distance $1-K\left(x_{k}, v_{i}\right)=1-\exp \left(-\left\|x_{k}-v_{i}\right\|^{2} / \sigma^{2}\right)$ and the kernel based objective function was modified as

$$
\begin{aligned}
J_{M}= & \sum_{i=1}^{c} \sum_{k=1}^{N} \mu_{i k}^{p}\left(1-K\left(x_{k}, v_{i}\right)\right) \\
& +\alpha \sum_{i=1}^{c} \sum_{k=1}^{N} \mu_{i k}^{p}\left(1-K\left(\tilde{x}_{k}, v_{i}\right)\right) .
\end{aligned}
$$

The necessary conditions for minimizing objective function are given:

$$
\begin{aligned}
& \mu_{i k} \\
& =\frac{\left(1-K\left(x_{k}, v_{i}\right)\right)+\alpha\left(1-K\left(\tilde{x}_{k}, v_{i}\right)\right)^{-1 /(p-1)}}{\sum_{j=1}^{c}\left(\left(1-K\left(x_{k}, v_{j}\right)\right)+\alpha\left(1-K\left(\tilde{x}_{k}, v_{j}\right)\right)\right)^{-1 /(p-1)}} \\
& v_{i}=\frac{\sum_{k=1}^{N} \mu_{i k}^{p}\left(K\left(x_{k}, v_{i}\right) x_{k}+\alpha K\left(\widetilde{x}_{k}, v_{i}\right) \tilde{x}_{k}\right)}{\sum_{k=1}^{N} \mu_{i k}^{p}\left(K\left(x_{k}, v_{i}\right)+\alpha K\left(\tilde{x}_{k}, v_{i}\right)\right)} .
\end{aligned}
$$

These algorithms with mean and median filter with Gaussian kernel induced distance are named as KFCM_S1 and KFCM_S2. This method can also be used to improve the performance of other FCM-like algorithms based on adding some type of penalty terms to the original FCM objective function.

\section{FCM Based Methods with Noise Robustness}

This section gives the mathematical analysis of FCM based methods for the segmentation of brain MR images with robustness to noise level in brain MR images.

6.1. Improved FCM Segmentation (IFS). Shen et al. [26] proposed the improved fuzzy $c$-means segmentation (IFS) algorithm to overcome noise effects in MR images. Instead of modifying the objective function of traditional FCM algorithm, it improves the similarity measurement of the pixel intensity and the cluster center by considering neighbourhood attraction. The objective function is expressed by

$$
J=\sum_{i=1}^{c} \sum_{k=1}^{N} \mu_{i k}^{p} d\left(x_{k}, v_{i}\right)
$$

where $d\left(x_{k}, v_{i}\right)$ is a similarity measurement between the pixel intensity and the cluster centers and is defined as follows:

$$
d\left(x_{k}, v_{i}\right)=\left\|x_{k}-v_{i}\right\|^{2}\left(1-\lambda H_{i k}-\xi F_{i k}\right)
$$

where $\lambda$ and $\xi$ adjust the degree of attraction and have a magnitude between 0 and 1 . Here, $H_{i k}$ is called feature attraction and $F_{i k}$ is called distance attraction given by

$$
\begin{gathered}
H_{i k}=\frac{\sum_{j=1}^{S} \mu_{i j} g_{k j}}{\sum_{j=1}^{S} g_{k j}}, \\
F_{i k}=\frac{\sum_{j=1}^{S} \mu_{i j}^{2} q_{k j}^{2}}{\sum_{j=1}^{S} q_{k j}^{2}}
\end{gathered}
$$

and, here, $g_{k j}=\left\|x_{k}-x_{j}\right\|$ is the intensity difference between study $k$ th pixel and its neighbouring $j$ th pixel and $q_{k j}$ is the relation location between $k$ th pixel and its neighbouring $j$ th pixel. $S$ is the number of neighbouring pixels and $\mu_{i j}$ is the membership of neighbouring $j$ th pixel to the $i$ th cluster. The bias field is estimated by the following equation:

$$
\beta_{k}=x_{k}-p_{k}-\frac{\sum_{k=1}^{N}\left(x_{k}-p_{k}\right)}{\sum_{i=1}^{c} \mu_{i k}^{p} M^{2} \sum_{k=1}^{N}\left(\sum_{i=1}^{c} \mu_{i k}^{p} M^{2}\right)},
$$

where $p_{k}=\sum_{i=1}^{c} \mu_{i k}^{p} M^{2} v_{i} / \sum_{i=1}^{c} \mu_{i k}^{p} M^{2}$ and $M=1-\lambda H_{i k}-$ $\xi F_{i k}$. The objective function is minimized with updates for membership value and cluster given as

$$
\begin{aligned}
\mu_{i k} & =\frac{1}{\sum_{j=1}^{c}\left(d\left(x_{k}, v_{i}\right) / d\left(x_{k}, v_{j}\right)\right)^{2 /(p-1)}}, \\
v_{i} & =\frac{\sum_{k=1}^{N} \mu_{i k}^{p}\left(x_{k}-\beta_{k}\right)}{\sum_{k=1}^{N} \mu_{i k}^{p}} .
\end{aligned}
$$

If a pixel has a very similar intensity to one of its neighbours, the attraction between them is stronger than the attraction between the pixel and another neighbour with 
different intensities. A spatially closer neighbouring pixel has a stronger attraction than a neighbour which is spatially distant. Segmentation using IFCM is decided by the pixel itself and by its neighbouring pixels which improves the segmentation results.

6.2. Fast Generalized FCM (FGFCM) Algorithm. FCM_S1 and FCM_S2, proposed in [21] as two extensions to FCM_S, have yielded effective segmentation for images, but both still lack enough robustness to noise and outliers in absence of prior knowledge of the noise. Parameter $\alpha$ is used to achieve balance between robustness to noise and effectiveness of preserving the details of the image; its selection has to be made by experimentation which is difficult. Also the time of segmenting an image is heavily dependent on the image size. Motivated by individual strengths of FCM_S1 and FCM_S2, Cai et al. [68] proposed a novel fast and robust FCM framework for image segmentation called FGFCM clustering algorithms incorporating local information. They introduced a novel factor $S_{k j}$ incorporating both the local spatial relationship (called $S_{s\lrcorner k j}$ ) and the local gray-level relationship (called $S_{g k j}$ ) to replace parameter $\alpha$ and make it play a more important role in clustering. Its definition is given by

$$
S_{k j}= \begin{cases}S_{s-k j} \times S_{g_{-} k j}, & j \neq k \\ 0, & j=k,\end{cases}
$$

where the $k$ th pixel is the center of the local window (e.g., $3 \times 3$ ) and $j$ th pixels are the set of the neighbours falling into a window around the $i$ th pixel given by

$$
S_{s, k j}=\exp \left(\frac{-\max \left(\left|p_{j}-p_{k}\right|,\left|q_{j}-q_{k}\right|\right)}{\lambda_{s}}\right),
$$

where $\left(p_{k}, q_{k}\right)$ is a spatial coordinate of the $k$ th pixel, $\lambda_{s}$ denotes the scale factor of the spread of $S_{s \_k j}$, determining its change characteristic, and $S_{s_{-} k j}$ reflects the damping extent of the neighbours with the spatial distances from the central pixel. The local gray-level similarity measure $S_{g_{-} k j}$ is given by

$$
S_{g \_k j}=\exp \left(\frac{-\left\|x_{k}-x_{j}\right\|^{2}}{\lambda_{g} \sigma_{g-k}^{2}}\right)
$$

where $x_{k}$ is gray value of the central pixel within a special window, $x_{j}$ is gray value of the $j$ th pixels in the same window, and $\lambda_{g}$ denotes the global scale factor of the spread of $S_{g \_j}$. The function of the local density surrounding the central pixel $\sigma_{g_{-} k}$ is given by

$$
\sigma_{g\lrcorner k}=\operatorname{sqrt}\left(\frac{\sum_{j \in N_{k}}\left\|x_{j}-x_{k}\right\|^{2}}{N_{R}}\right) \text {. }
$$

The value of parameter $\sigma_{g k}$ reflects gray value homogeneity degree of the local window. The smaller its value is, the more homogeneous the local window is, and vice versa. $\sigma_{g_{-} k}$ can change automatically with different gray-levels of the pixels over an image and thus reflects the damping extent in gray values. FGFCM clustering algorithm incorporates local spatial and gray-level information into its objective function which is given by

$$
J=\sum_{i=1}^{c} \sum_{k=1}^{q} \gamma_{k} \mu_{i k}^{p}\left(\xi_{k}-v_{i}\right)^{2}
$$

where $v_{i}$ represents the prototype of the $i$ th cluster and represents the fuzzy membership of gray value $k$ with respect to cluster $i . \gamma_{k}$ is the number of the pixels having the gray value equal to $k$, where $k=1,2, \ldots, q$ and $\sum_{k=1}^{q} \gamma_{k}=N$, and $q$ denotes the number of the gray-levels of the given image which is generally much smaller than $N$. The new generated image is computed in terms of

$$
\xi_{k}=\frac{\sum_{j \in N_{k}} S_{k j} x_{j}}{\sum_{j \in N_{k}} S_{k j}}
$$

where $x_{j}$ is gray value of the neighbours of $x_{k}$ (window center), $N_{k}$ is set of neighbours falling in the local window, and $S_{k j}$ is local similarity measure between the $k$ th pixel and the $j$ th pixel. $S_{k j}$ can be considered as the weight of the $j$ th pixel and $\xi_{k}$ can be considered as the $k$ th pixel of the linearly weighted summed image. The partition matrix and cluster centroid are updated by

$$
\begin{aligned}
\mu_{i k} & =\frac{\left(\xi_{k}-v_{i}\right)^{-2 /(p-1)}}{\sum_{j=1}^{c}\left(\left(\xi_{k}-v_{j}\right)^{-2 /(p-1)}\right)} \\
v_{i} & =\frac{\sum_{k=1}^{q} \gamma_{k} \mu_{i k}^{p} \xi_{k}}{\sum_{k=1}^{q} \gamma_{k} \mu_{i k}^{p}}
\end{aligned}
$$

FGFCM introduces a new factor $S_{k j}$ as a local (spatial and gray) similarity measure with robustness to noise and detailpreserving for image and removes the empirically adjusted parameter $\alpha$. FGFCM produces fast clustering for given image, which is attributed to its dependence only on the number of the gray-levels $q$ rather than the size $N$ of the image, which reduces its time complexity of clustering.

6.3. A Gaussian Kernel Based FCM (GKFCM). Yang and Tsai [27] modified objective function given by Chen and Zhang [21] to make it independent of parameter $\alpha$. The modified objective function is given by

$$
\begin{aligned}
J= & \sum_{i=1}^{c} \sum_{k=1}^{N} \mu_{i k}^{p}\left(1-K\left(x_{k}, v_{i}\right)\right) \\
& +\sum_{i=1}^{c} \sum_{k=1}^{N} \eta_{i} \mu_{i k}^{p}\left(1-K\left(\tilde{x}_{k}, v_{i}\right)\right),
\end{aligned}
$$

where $K(x, y)=\exp \left(-\|x-y\|^{2} / \sigma^{2}\right), \sigma^{2}=\sum_{k=1}^{N}\left(\left\|x_{k}-\bar{x}\right\|^{2} / \eta_{i}\right)$ with $\bar{x}=\sum_{j=1}^{N}\left(x_{k} / N\right)$, and $\eta_{i}=\min _{i^{\prime} \neq i}\left(1-K\left(v_{i}^{\prime}, v_{i}\right)\right) / \max _{j}(1-$ $\left.K\left(v_{j}, \bar{x}\right)\right)$. 
The membership function and cluster prototype are updated as follows:

$$
\begin{aligned}
& \mu_{i k} \\
& =\frac{\left(\left(1-K\left(x_{k}, v_{i}\right)\right)+\eta_{i}\left(1-K\left(\tilde{x}, v_{i}\right)\right)\right)^{-1 /(p-1)}}{\sum_{j=1}^{c}\left(\left(1-K\left(x_{k}, v_{j}\right)\right)+\eta_{i}\left(1-K\left(\tilde{x}, v_{j}\right)\right)\right)^{-1 /(p-1)}} \\
& v_{i}=\frac{\sum_{k=1}^{N} \mu_{i k}^{p}\left(K\left(x_{k}, v_{i}\right) x_{k}+\eta_{i} K\left(\tilde{x}_{k}, v_{i}\right) \tilde{x}_{k}\right)}{\sum_{k=1}^{N} \mu_{i k}^{p}\left(K\left(x_{k}, v_{i}\right)+\eta_{i} K\left(\tilde{x}_{k}, v_{i}\right)\right)} .
\end{aligned}
$$

GKFCM automatically learn the parameters by the prototype-driven learning scheme. The results of GKFCM are more robust to noise and outliers than BCFCM, KFCM_S1, and KFCM_S2 especially in segmenting MR images.

\subsection{A Modified FCM Method Using Multiscale Fuzzy c-Means} (MsFCM). Wang and Fei [44] proposed multiscale fuzzy $c$ means (MsFCM) algorithm for the classification of brain MR images which performs classification from the coarsest to the finest scale, that is, the original image. The classification result at a coarser level $t+1$ was used to initialize the classification at a higher scale level $t$. The final classification is the result at scale level 0 . During the classification processing at level $t+1$, the pixels with the highest membership above a threshold are identified and assigned to the corresponding class. These pixels are labelled as training data for the next level $t$. The objective function of the MsFCM at level $t$ is given by

$$
\begin{aligned}
J= & \sum_{i=1}^{c} \sum_{k=1}^{N} \mu_{i k}^{p}\left\|x_{k}-v_{i}\right\|^{2} \\
& +\frac{\alpha}{N_{R}} \sum_{i=1}^{c} \sum_{k=1}^{N} \mu_{i k}^{p}\left(\sum_{y_{r} \in N_{k}}\left\|y_{r}-v_{i}\right\|^{2}\right) \\
& +\beta \sum_{i=1}^{c} \sum_{k=1}^{N}\left(\mu_{i k}-\mu_{i k}^{\prime}\right)^{p}\left\|x_{k}-v_{i}\right\|^{2}
\end{aligned}
$$

and, here, $\alpha$ and $\beta$ are scaling factors. $\mu_{i k}^{\prime}$ is the membership obtained from the classification in the previous scale and is determined by

$$
\mu_{i k}^{\prime}= \begin{cases}\mu_{i k}^{\prime}, & \text { if } \max \left(\mu_{i k}^{t+1}\right)>K \\ 0, & \text { otherwise, }\end{cases}
$$

where $K$ is the threshold to determine the pixels with a known class in the next scale classification and is set as 0.85 . The partition matrix and class centers are updated by

$$
\begin{aligned}
\mu_{i k} & =\frac{1+\beta \sum_{j=1}^{c}\left(\left(\mu_{i k}^{\prime} d_{i k}^{2}-\mu_{i j}^{\prime} d_{i k}^{2}\right) /\left((1+\beta) d_{i k}^{2}+\left(\alpha / N_{R}\right)\left(\sum_{x_{r} \in N_{k}} d_{i k}^{2}\right)\right)\right)}{\sum_{j=1}^{c}\left(\left((1+\beta) d_{i k}^{2}+\left(\alpha / N_{R}\right)\left(\sum_{x_{r} \in N_{k}} d_{j k}^{2}\right)\right) /\left((1+\beta) d_{j k}^{2}+\left(\alpha / N_{R}\right)\left(\sum_{x_{r} \in N_{k}} d_{j k}^{2}\right)\right)\right)} \\
v_{i} & =\frac{\sum_{k=1}^{N} \mu_{i k}^{2}\left(x_{k}+\left(\alpha / N_{R}\right) \sum_{x_{r} \in N_{k}} x_{r}\right)+\beta \sum_{k=1}^{N}\left(\mu_{i k}-\mu_{i k}^{\prime}\right)^{2} x_{k}}{(1+\alpha) \sum_{k=1}^{N} \mu_{i k}^{2}+\beta \sum_{k=1}^{N}\left(\mu_{i k}-\mu_{i k}^{\prime}\right)^{2}} .
\end{aligned}
$$

The multiscale scheme improves the speed of the classification and robustness. The centroids of the initial classes from the coarser image improve the convergence of the classification algorithm. A pixel with a high probability of belonging to one class in the coarse image is expected to belong to the same class in the next fine image. Threshold $K$ is used to select these pixels at the coarse level and thus to constrain the classification in the next level. A smaller threshold means more reliable classification in the coarse image. This threshold depends on noise levels and preprocessing such as diffusion filtering. A smaller threshold is expected for images with better image quality.

\section{FCM Methods with Noise Robustness and Intensity Inhomogeneity Correction}

This section includes the mathematical analysis of FCM based method for the segmentation of brain MR images with intensity inhomogeneity correction and robustness to noise.
7.1. A Framework with Modified Fast FCM (MFCM) Algorithm. Ji et al. [15] proposed a new automated method to determine the initial value of centroid and also an adaptive method to incorporate the local spatial continuity in the segmentation of brain MR image. The objective function for modified FCM algorithm is given by

$$
\begin{aligned}
J= & \sum_{i=1}^{c} \sum_{k=1}^{N}\left[(1-\alpha) \mu_{i k}^{p}\left(y_{k}-b_{k}-v_{i}\right)^{2}\right. \\
& \left.+\alpha \sum_{r \in N_{k}} \mu_{i k}^{p} \omega_{k r}\left(y_{r}-b_{r}-v_{i}\right)^{2}\right]+\sum_{k=1}^{N} \alpha_{k} \sum_{i=1}^{c} \mu_{i k}(1 \\
& \left.-\mu_{i k}^{p-1}\right)
\end{aligned}
$$

where $\omega_{k r}$ is the weight of pixels in the neighbourhood centered at $k$ th pixel. $\alpha_{k}$ is the control parameter of the term which rewards the crispness membership degrees. In this algorithm the initialization of centroid was done automatically using histon and adaptive method was proposed to incorporate the local spatial continuity to overcome the noise 
effectively and prevent the edges from blurring. The intensity inhomogeneity is estimated by linear combination of the set of basis functions and regularization term is added in objective function to reduce the number of iteration steps. The partition matrix and cluster centroid and bias field are updated as

$$
\begin{aligned}
& \mu_{i k}=\left(\sum_{j=1}^{c}\left(\frac{(1-\alpha) d_{k i}+\alpha \sum_{r \in N_{k}} \omega_{k r} d_{r i}-\alpha_{k}}{(1-\alpha) d_{k j}+\alpha \sum_{r \in N_{k}} \omega_{k r} d_{r j}-\alpha_{k}}\right)\right)^{-1} \\
& v_{i} \\
& =\frac{\sum_{k=1}^{N} \mu_{i k}^{p}(1-\alpha)\left(y_{k}-b_{k}\right)+\alpha \sum_{r \in N_{k}} \omega_{k r}\left(y_{r}-b_{r}\right)}{\sum_{k=1}^{N} \mu_{i k}^{p}} \\
& b_{k}=\sum_{k=1}^{M} \bar{\omega}_{k} g_{k}(k)=\bar{\omega}^{T} G(k),
\end{aligned}
$$

where $d_{k i}=\left(y_{k}-b_{k}-v_{i}\right)^{2}, \bar{\omega}=\left(\bar{\omega}_{1}, \bar{\omega}_{2}, \ldots, \bar{\omega}_{M}\right)^{T}$, and $G(k)=\left(g_{1}(k), g_{2}(k), \ldots, g_{M}(k)\right)^{T}$, and here $\bar{\omega}_{k} \in \mathfrak{R}, k=$ $1,2, \ldots, M$, are the combination coefficients. The orthogonal polynomials are used as the basis functions which satisfy the condition given as follows:

$$
\int_{\Omega} g_{i}(x) g_{j}(x)=\delta_{i j}
$$

where $\delta_{i j}=0, i \neq j, \delta_{i j}=1, i=j, \bar{\omega}=A^{-1} B, A=$ $\sum_{k \in \Omega} G(k) G(k)^{T} \sum_{i=1}^{c} \mu_{i k}^{p}$, and $B=\sum_{k \in \Omega} G(k) \sum_{i=1}^{c} \mu_{i k}^{p}\left(y_{k}-\right.$ $\left.v_{i}\right)$. $A$ is $M \times M$ is matrix and $B$ is $M \times 1$ matrix with $M$ being the number of the basis functions. MFCM method overcomes the three major artifacts of brain images (intensity inhomogeneity, noise, and partial volume effect) at the same time. The initial values of the centroids are determined automatically. Then, an adaptive method to incorporate the local spatial continuity is used to overcome the noise effectively and prevent the edge from blurring. The intensity inhomogeneity is estimated by a linear combination of a set of basis functions. A regularization term is added to reduce the iteration steps and accelerate the algorithm. The weights of the regularization terms are all automatically computed to avoid the manually tuned parameter.

7.2. Modified Robust FCM Algorithm with Weighted Bias Estimation (MRFCM-wBE). Ramathilagam et al. [19] proposed a modified robust fuzzy $c$-means algorithm with special weighted bias estimation (MRFCM-wBE) for segmentation of brain MR images. To reduce the number of iterations, the proposed robust algorithm initializes the centroid using dist-max initialization algorithm before the execution of algorithm iteratively. The objective function of MRFCM-wBE is given by

$$
J=\sum_{i=1}^{c} \sum_{k=1}^{N} \mu_{i k}^{p}\left\|y_{k}-\omega_{k} \beta_{k}-v_{i}\right\|^{2}+\tilde{x}\left(1+\sum_{i=1}^{c} \mu_{i k}^{p}\right),
$$

where $\tilde{x}=\left(\sum_{k} y_{k}\right) / N$ and $\omega_{k} \in(0,1)$ is the weight of $k$ th pixel. The partition matrix, centroid cluster, and bias field are updated as follows:

$$
\begin{aligned}
\mu_{i k}^{*} & =\sum_{j=1}^{c}\left(\left(\frac{\left\|y_{k}-\omega_{k} \beta_{k}-v_{i}\right\|^{2}+\tilde{x}}{\left\|y_{k}-\omega_{k} \beta_{k}-v_{j}\right\|^{2}+\tilde{x}}\right)^{1 /(p-1)}\right)^{-1} \\
v_{i}^{*} & =\frac{\sum_{k=1}^{N} \mu_{i k}^{p}\left(y_{k}-\omega_{k} \beta_{k}\right)}{\sum_{k=1}^{N} \mu_{i k}^{p}} \\
\beta_{k}^{*} & =\frac{1}{\omega_{k}}\left(y_{k}-\frac{\sum_{i=1}^{c} \mu_{i k}^{p} v_{i}}{\sum_{i=1}^{c} \mu_{i k}^{p}}\right) .
\end{aligned}
$$

Experimental results of MRFCM-wBE indicate that the algorithm is more robust to the noises and faster than many other segmentation algorithms.

7.3. Efficient Inhomogeneity Compensation Using FCM Clustering Models. The compensation of intensity inhomogeneity artifacts is a computationally costly problem, which demands highly efficient design and implementation. Szilágyi et al. [69] demonstrated intensity inhomogeneity compensation on a single-channel intensity image via $c$-means clustering models. The operations performed during the iterations of the alternating optimization ( $\mathrm{AO})$ scheme are separated into globally working ones and locally applied ones, and their execution is optimized according to their necessities. Global criteria are applied to gray intensities instead of individual pixels, which makes a drastic reduction of the computational load. This formulation and improved clustering models are combined with multistage INU compensation to obtain high accuracy and efficiency. The objective function of efficient FCM method is given by

$$
J_{\mathrm{FCM}_{-} q b}=\sum_{i=1}^{c} \sum_{l \in \Omega^{(t)}} h_{l}^{t} \mu_{i l}^{p}\left(1-v_{i}\right)^{2}
$$

and, here, $h_{l}^{t}$ is the number of pixels for which the compensated intensity in iteration $t$ satisfies $y_{k}-b_{k}=1 . \Omega^{(t)}$ is the range of possible values of $y_{k}-b_{k}$ and $\sum_{l \in \Omega^{(t)}} h_{l}^{(t)}=n$. And hence $h_{l}^{t}$ with $l \in \Omega^{(t)}$ represents the intensity histogram of the compensated image in iteration $t$. Thus, for any compensated intensity $l \in \Omega^{(t)}$ and any cluster indexed $i=1,2, \ldots, c$, the partition matrix and centroid cluster are updated as follows:

$$
\begin{aligned}
\mu_{i l} & =\frac{\left(l-v_{i}\right)^{-2 /(p-1)}}{\sum_{j=1}^{c}\left(1-v_{j}\right)^{-2 /(p-1)}} \\
v_{i} & =\frac{\sum_{l \in \Omega^{(t)}} h_{l}^{t} \mu_{i l}^{p} l}{\sum_{l \in \Omega^{(t)}} h_{l}^{t} \mu_{i l}^{p}} .
\end{aligned}
$$

For any $l \in \Omega^{(t)}$, auxiliary variables are defined and organized in look-up table as $q_{l}=\sum_{i=1}^{c} \mu_{i l}^{p} v_{i} / \sum_{i=1}^{c} \mu_{i l}^{p}$. For any pixel with index $k=1, \ldots, n$, the bias field is estimated as

$$
b_{k}=y_{k}-q_{l_{k}}
$$

with $l_{k}=y_{k}-b_{k}^{t-1}$. 


\section{Validation Methods}

Quantitative evaluation is essential for objective comparison of the results of different correction methods. Commonly used metrics for inhomogeneity correction through segmentation are as follows:

(1) Segmentation accuracy (SA) used in [14] is defined as

$\mathrm{SA}=\left(\frac{(\text { Number } \text { of Correctly Classified Pixels })}{(\text { Total Number of Pixels })}\right)$

$\times 100$.

(2) Three evaluation parameters used in [26, 44] are defined as follows:

(a) Undersegmentation representing the \% of negative false segmentation, $N_{f p} / N_{n}$.

(b) Oversegmentation representing the \% of positive false segmentation, $N_{f n} / N_{p}$.

(c) Incorrect segmentation representing the total \% of false segmentation, $\left(N_{f n}+N_{f p}\right) / N$,

where $N_{f p}$ is the number of pixels that do not belong to a cluster and are segmented into the cluster. $N_{f n}$ is the number of pixels that belong to a cluster and are not segmented into the cluster. $N_{p}$ is the number of all pixels that belong to a cluster and $N_{n}$ is the total number of pixels that do not belong to a cluster.

(3) Jaccard similarity and the Dice coefficient estimate the segmentation of voxels of one segmented tissue. The Jaccard similarity, used in [15-17], is defined as the ratio between intersection and union of two sets and representing the obtained and gold standard segmentations, respectively:

$$
J\left(S_{1}, S_{2}\right)=\frac{S_{1} \cap S_{2}}{S_{1} \cup S_{2}} .
$$

The authors also used the Dice coefficient, which is a special case of the index, defined as

$$
\kappa\left(S_{1}, S_{2}\right)=\frac{2\left|S_{1} \cap S_{2}\right|}{\left|S_{1}\right|+\left|S_{2}\right|} .
$$

In comparison to the Dice coefficient, the Jaccard similarity is more sensitive when sets are more similar. Jaccard similarity is referred to as segmentation measure or comparison score in $[21,68]$.

(4) The silhouette width $S(i)$ of the object $i$ used in [19] is obtained using the equation

$$
S(i)=\frac{b(i)-a(i)}{\max b(i), a(i)}
$$

In the above equation $a(i)$ is the average distance between the $i$ th data and all other data in the cluster. $b(i)$ is the smallest average distance between the $i$ th data and all other data of other clusters. All these segmentation based measures provide quantitative information on segmentation accuracy, assuming that gold standard segmentations are available.

\section{Dataset Used for Validation}

An objective validation method may require a ground truth, based on strong prior knowledge about the real structure of the object of interest. For datasets acquired in clinical situation, this ground truth is usually incomplete, leading to a subjective quality assessment. Consequently, other solutions have been proposed for validation purpose, leading to the use of numerical data, either synthetic or simulated, or the acquisition of images of physical phantoms with known characteristics.

(1) Numerical datasets: Numerical datasets are commonly used for validation because of the ideal prior knowledge they provide. They allow both qualitative and quantitative evaluation. Typical numerical datasets are either synthetic or simulated.

(2) Synthetic datasets: These datasets carry no realistic anatomical or physical information. They are usually used as a first step for objectively evaluating an image processing method with their characteristics being already known. Since a synthetic dataset does not have realistic behaviour, simulated datasets have become necessary. With the increase in computational power, such realistic datasets can be simulated. An MRI simulator and simulated brain datasets are available from the McConnell Brain Imaging Centre at the Montreal Neurological Institute and Hospital [13].

(3) Real datasets: The Internet Brain Segmentation Repository (IBSR) [70] is a World Wide Web resource providing access to magnetic resonance brain image data and segmentation results contributed and utilized by researchers.

A comparison of these algorithmic approaches for brain MR image segmentation with intensity inhomogeneity correction using fuzzy clustering in terms of advantages and limitations is summarized in Table 1.

\section{Conclusion}

In this paper, a comprehensive review of FCM clustering algorithms for the segmentation of MR brain images with intensity inhomogeneity corrections and noise robustness is presented. The algorithms are analysed according to various features like modification of standard fuzzy objective function and updating of fuzzy membership function and cluster center. A number of important issues have been emphasized, like algorithmic parameter selection, computational complexity, and noise robustness, indicating that intensity inhomogeneity correction as well as noise removal is still a challenging task. Because of intensity inhomogeneity, noise, 


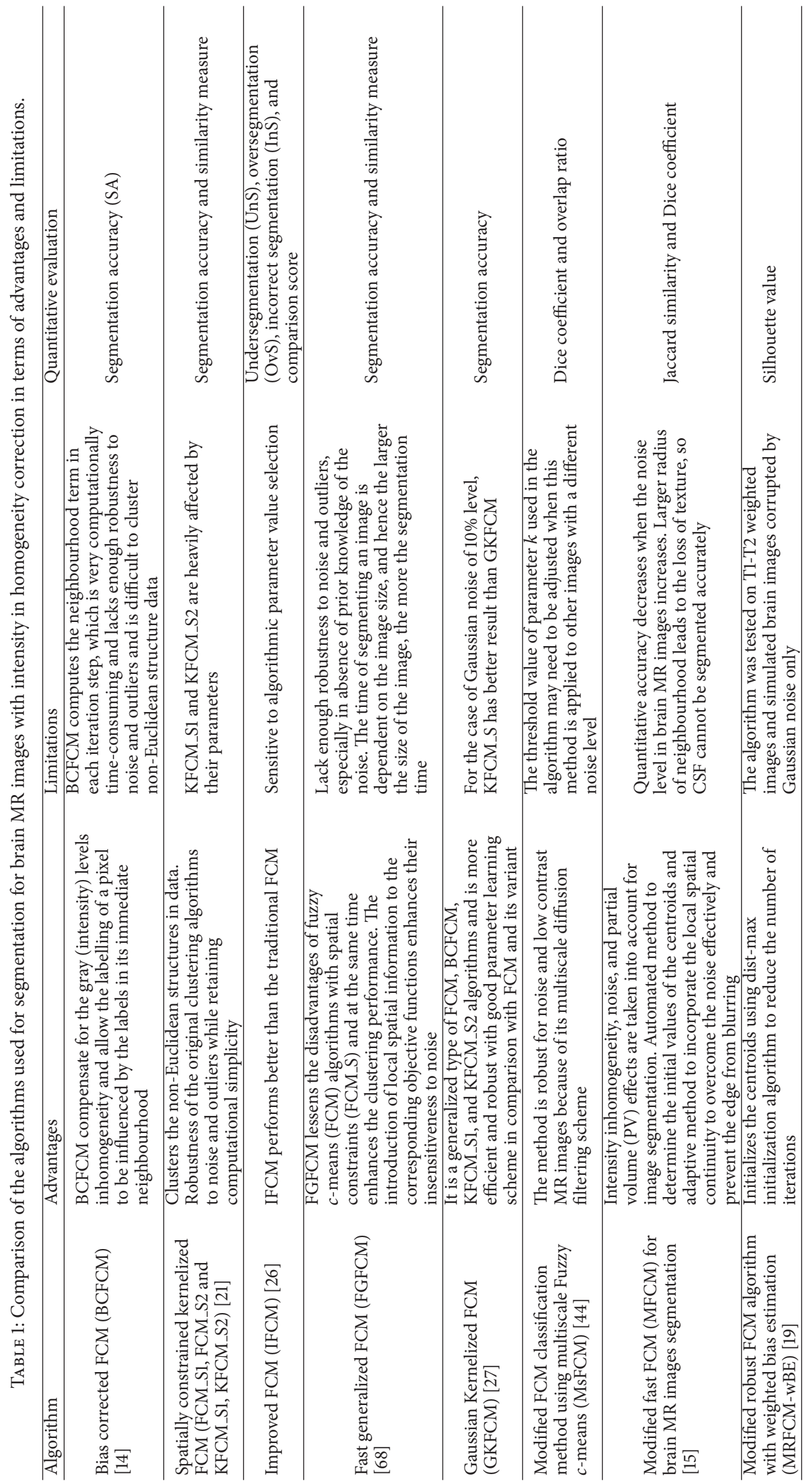


and weak boundaries, magnetic resonance brain segmentation is still a challenging area of research and there is a need for future research to improve the accuracy, precision, and speed of segmentation methods.

\section{Competing Interests}

The authors declare that there is no conflict of interests regarding the publication of this paper.

\section{References}

[1] T. L. Huntsherger, C. L. Jacobs, and R. L. Cannon, "Iterative fuzzy image segmentation," Pattern Recognition, vol. 18, no. 2, pp. 131-138, 1985.

[2] M. Trivedi and J. C. Bezdek, "Low level segmentation of aerial images with fuzzy clustering," IEEE Transaction on System, Man, Cybernate, vol. 16, no. 4, pp. 589-598, 1986.

[3] F. Masulli and A. Schenone, "A fuzzy clustering based segmentation system as support to diagnosis in medical imaging," Artificial Intelligence in Medicine, vol. 16, no. 2, pp. 129-147, 1999.

[4] A. Simmons, P. S. Tofts, G. J. Barker, and S. R. Arridge, "Sources of intensity nonuniformity in spin echo images at 1.5 T," Magnetic Resonance in Medicine, vol. 32, no. 1, pp. 121-128, 1994.

[5] J. G. Sled and G. B. Pike, "Understanding intensity non-uniformity in MRI," in Medical Image Computing and ComputerAssisted Interventation-MICCAI '98: First International Conference Cambridge, MA, USA, October 11-13, 1998 Proceedings, vol. 1496 of Lecture Notes in Computer Science, pp. 614-622, Springer, Berlin, Germany, 1998.

[6] R. P. Velthuizen, J. J. Heine, A. B. Cantor, H. Lin, L. M. Fletcher, and L. P. Clarke, "Review and evaluation of MRI nonuniformity corrections for brain tumor response measurements," Medical Physics, vol. 25, no. 9, pp. 1655-1666, 1998.

[7] J. B. Arnold, J.-S. Liow, K. A. Schaper et al., "Qualitative and quantitative evaluation of six algorithms for correcting intensity nonuniformity effects," NeuroImage, vol. 13, no. 5, pp. 931-943, 2001.

[8] Z. Hou, "A review on MR image intensity inhomogeneity correction," International Journal of Biomedical Imaging, vol. 2006, Article ID 49515, 11 pages, 2006.

[9] B. Belaroussi, J. Milles, S. Carme, Y. M. Zhu, and H. BenoitCattin, "Intensity non-uniformity correction in MRI: existing methods and their validation," Medical Image Analysis, vol. 10, no. 2, pp. 234-246, 2006.

[10] U. Vovk, F. Pernuš, and B. Likar, "A review of methods for correction of intensity inhomogeneity in MRI," IEEE Transactions on Medical Imaging, vol. 26, no. 3, pp. 405-421, 2007.

[11] M. A. Balafar, A. R. Ramli, M. I. Saripan, and S. Mashohor, "Review of brain MRI image segmentation methods," Artificial Intelligence Review, vol. 33, no. 3, pp. 261-274, 2010.

[12] W. M. Wells III, W. E. L. Crimson, R. Kikinis, and F. A. Jolesz, "Adaptive segmentation of mri data," IEEE Transactions on Medical Imaging, vol. 15, no. 4, pp. 429-442, 1996.

[13] Brainweb, http://www.bic.mni.mcgill.ca/brainweb/.

[14] M. N. Ahmed, S. M. Yamany, N. Mohamed, A. A. Farag, and T. Moriarty, "A modified fuzzy c-means algorithm for bias field estimation and segmentation of MRI data," IEEE Transactions on Medical Imaging, vol. 21, no. 3, pp. 193-199, 2002.
[15] Z.-X. Ji, Q.-S. Sun, and D.-S. Xia, "A framework with modified fast FCM for brain MR images segmentation," Pattern Recognition, vol. 44, no. 5, pp. 999-1013, 2011.

[16] Z.-X. Ji, Q.-S. Sun, and D.-S. Xia, "A modified possibilistic fuzzy c-means clustering algorithm for bias field estimation and segmentation of brain MR image," Computerized Medical Imaging and Graphics, vol. 35, no. 5, pp. 383-397, 2011.

[17] Z. Ji, Q. Sun, Y. Xia, Q. Chen, D. Xia, and D. Feng, "Generalized rough fuzzy c-means algorithm for brain MR image segmentation," Computer Methods and Programs in Biomedicine, vol. 108, no. 2, pp. 644-655, 2012.

[18] S. R. Kannan, "A new segmentation system for brain MR images based on fuzzy techniques," Applied Soft Computing Journal, vol. 8, no. 4, pp. 1599-1606, 2008.

[19] S. Ramathilagam, R. Pandiyarajan, A. Sathya, R. Devi, and S. R. Kannan, "Modified fuzzy c-means algorithm for segmentation of T1-T2-weighted brain MRI," Journal of Computational and Applied Mathematics, vol. 235, no. 6, pp. 1578-1586, 2011.

[20] D. L. Pham and J. L. Prince, "An adaptive fuzzy C-means algorithm for image segmentation in the presence of intensity inhomogeneities," Pattern Recognition Letters, vol. 20, no. 1, pp. 57-68, 1999.

[21] S. Chen and D. Zhang, "Robust image segmentation using FCM with spatial constraints based on new kernel-induced distance measure," IEEE Transactions on Systems, Man, and Cybernetics, Part B: Cybernetics, vol. 34, no. 4, pp. 1907-1916, 2004.

[22] Y. A. Tolias and S. M. Panas, "Image segmentation by a fuzzy clustering algorithm using adaptive spatially constrained membership functions," IEEE Transactions on Systems, Man, and Cybernetics-Part A: Systems and Humans., vol. 28, no. 3, pp. 359-369, 1998.

[23] Y. A. Tolias and S. M. Panas, "On applying spatial constraints in fuzzy image clustering using a fuzzy rule-based system," IEEE Signal Processing Letters, vol. 5, no. 10, pp. 245-247, 1998.

[24] L. Szilagyi, Z. Benyo, S. M. Szilagyii, and H. S. Adam, "MR brain image segmentation using an enhanced fuzzy C-means algorithm," in Proceedings of the 25th Annual International Conference of the IEEE Engineering in Medicine and Biology Society, vol. 1, pp. 724-726, IEEE, Buenos Aires, Argentina, September 2003.

[25] J. Liu, J. K. Udupa, D. Odhner, D. Hackney, and G. Moonis, "A system for brain tumor volume estimation via MR imaging and fuzzy connectedness," Computerized Medical Imaging and Graphics, vol. 29, no. 1, pp. 21-34, 2005.

[26] S. Shen, W. Sandham, M. Granat, and A. Sterr, "MRI fuzzy segmentation of brain tissue using neighborhood attraction with neural-network optimization," IEEE Transactions on Information Technology in Biomedicine, vol. 9, no. 3, pp. 459-467, 2005.

[27] M.-S. Yang and H.-S. Tsai, "A Gaussian kernel-based fuzzy cmeans algorithm with a spatial bias correction," Pattern Recognition Letters, vol. 29, no. 12, pp. 1713-1725, 2008.

[28] M. S. Atkins and B. T. Mackiewich, "Fully automatic segmentation of the brain in MRI," IEEE Transactions on Medical Imaging, vol. 17, no. 1, pp. 98-107, 1998.

[29] O. Colliot, O. Camara, and I. Bloch, "Integration of fuzzy spatial relations in deformable models-application to brain MRI segmentation," Pattern Recognition, vol. 39, no. 8, pp. 1401$1414,2006$.

[30] A. Zavaljevski, A. P. Dhawan, M. Gaskil, W. Ball, and J. D. Johnson, "Multi-level adaptive segmentation of multi-parameter MR 
brain images," Computerized Medical Imaging and Graphics, vol. 24, no. 2, pp. 87-98, 2000.

[31] J. J. Corso, E. Sharon, S. Dube, S. El-Saden, U. Sinha, and A. Yuille, "Efficient multilevel brain tumor segmentation with integrated bayesian model classification," IEEE Transactions on Medical Imaging, vol. 27, no. 5, pp. 629-640, 2008.

[32] R. Cárdenes, R. de Luis-García, and M. Bach-Cuadra, "A multidimensional segmentation evaluation for medical image data," Computer Methods and Programs in Biomedicine, vol. 96, no. 2, pp. 108-124, 2009.

[33] A. Akselrod-Ballin, M. Galun, J. M. Gomori et al., "Automatic segmentation and classification of multiple sclerosis in multichannel MRI," IEEE Transactions on Biomedical Engineering, vol. 56, no. 10, pp. 2461-2469, 2009.

[34] S. Datta and P. A. Narayana, "A comprehensive approach to the segmentation of multichannel three-dimensional MR brain images in multiple sclerosis," NeuroImage: Clinical, vol. 2, no. 1, pp. 184-196, 2013.

[35] S. Bricq, C. Collet, and J. P. Armspach, "Unifying framework for multimodal brain MRI segmentation based on Hidden Markov Chains," Medical Image Analysis, vol. 12, no. 6, pp. 639-652, 2008.

[36] A. R. Ferreira da Silva, "Bayesian mixture models of variable dimension for image segmentation," Computer Methods and Programs in Biomedicine, vol. 94, no. 1, pp. 1-14, 2009.

[37] B. Scherrer, F. Forbes, C. Garbay, and M. Dojat, "Distributed local MRF models for tissue and structure brain segmentation," IEEE Transactions on Medical Imaging, vol. 28, no. 8, pp. 1278$1295,2009$.

[38] J. Nie, Z. Xue, T. Liu et al., "Automated brain tumor segmentation using spatial accuracy-weighted hidden markov random field," Computerized Medical Imaging and Graphics, vol. 33, no. 6, pp. 431-441, 2009.

[39] A. Huang, R. Abugharbieh, and R. Tam, "A hybrid geometricstatistical deformable model for automated 3-D segmentation in brain MRI," IEEE Transactions on Biomedical Engineering, vol. 56, no. 7, pp. 1838-1848, 2009.

[40] L. Wang, Y. Chen, X. Pan, X. Hong, and D. Xia, "Level set segmentation of brain magnetic resonance images based on local Gaussian distribution fitting energy," Journal of Neuroscience Methods, vol. 188, no. 2, pp. 316-325, 2010.

[41] C. Li, R. Huang, Z. Ding, J. C. Gatenby, D. N. Metaxas, and J. C. Gore, "A level set method for image segmentation in the presence of intensity inhomogeneities with application to MRI," IEEE Transactions on Image Processing, vol. 20, no. 7, pp. 20072016, 2011.

[42] K. Thapaliya, J.-Y. Pyun, C.-S. Park, and G.-R. Kwon, "Level set method with automatic selective local statistics for brain tumor segmentation in MR images," Computerized Medical Imaging and Graphics, vol. 37, no. 7-8, pp. 522-537, 2013.

[43] Z. M. Wang, Y. C. Soh, Q. Song, and K. Sim, "Adaptive spatial information-theoretic clustering for image segmentation," Pattern Recognition, vol. 42, no. 9, pp. 2029-2044, 2009.

[44] H. Wang and B. Fei, "A modified fuzzy c-means classification method using a multiscale diffusion filtering scheme," Medical Image Analysis, vol. 13, no. 2, pp. 193-202, 2009.

[45] P. Maji and S. K. Pal, "Rough set based generalized fuzzy Cmeans algorithm and quantitative indices," IEEE Transactions on Systems, Man, and Cybernetics Part B: Cybernetics, vol. 37, no. 6, pp. 1529-1540, 2007.
[46] S. Mitra, W. Pedrycz, and B. Barman, "Shadowed c-means: integrating fuzzy and rough clustering," Pattern Recognition, vol. 43, no. 4, pp. 1282-1291, 2010.

[47] R. He, S. Datta, B. R. Sajja, and P. A. Narayana, "Generalized fuzzy clustering for segmentation of multi-spectral magnetic resonance images," Computerized Medical Imaging and Graphics, vol. 32, no. 5, pp. 353-366, 2008.

[48] P. Hore, L. O. Hall, D. B. Goldgof, Y. Gu, A. A. Maudsley, and A. Darkazanli, "A scalable framework for segmenting magnetic resonance images," Journal of Signal Processing Systems, vol. 54, no. 1-3, pp. 183-203, 2009.

[49] B. Caldairou, N. Passat, P. A. Habas, C. Studholme, and F. Rousseau, "A non-local fuzzy segmentation method: application to brain MRI," Pattern Recognition, vol. 44, no. 9, pp. 19161927, 2011.

[50] T. Chaira, "A novel intuitionistic fuzzy C means clustering algorithm and its application to medical images," Applied Soft Computing Journal, vol. 11, no. 2, pp. 1711-1717, 2011.

[51] Y. K. Dubey and M. M. Mushrif, "Segmentation of brain MR images using intuitionistic fuzzy clustering algorithm," in Proceedings of the 8th Indian Conference on Computer Vision, Graphics and Image Processing (ICVGIP '12), ACM, Mumbai, India, December 2012.

[52] P. Coupe, P. Yger, S. Prima, P. Hellier, C. Kervrann, and C. Barillot, "An optimized blockwise nonlocal means denoising filter for 3-D magnetic resonance images," IEEE Transactions on Medical Imaging, vol. 27, no. 4, pp. 425-441, 2008.

[53] H. Liu, C. Yang, N. Pan, E. Song, and R. Green, "Denoising 3D MR images by the enhanced non-local means filter for Rician noise," Magnetic Resonance Imaging, vol. 28, no. 10, pp. 14851496, 2010.

[54] J. V. Manjón, P. Coupé, L. Martí-Bonmatí, D. L. Collins, and M. Robles, "Adaptive non-local means denoising of MR images with spatially varying noise levels," Journal of Magnetic Resonance Imaging, vol. 31, no. 1, pp. 192-203, 2010.

[55] J. Hu, Y. Pu, X. Wu, Y. Zhang, and J. Zhou, "Improved DCT-based nonlocal means filter for MR images denoising," Computational and Mathematical Methods in Medicine, vol. 2012, Article ID 232685, 14 pages, 2012.

[56] P. Perona and J. Malik, "Scale-space and edge detection using anisotropic diffusion," IEEE Transactions on Pattern Analysis and Machine Intelligence, vol. 12, no. 7, pp. 629-639, 1990.

[57] C. Undeman and T. Lindeberg, "Fully automatic segmentation of mri brain images using probabilistic anisotropic diffusion and multi-scale watersheds," in Scale Space Methods in Computer Vision, vol. 2695 of Lecture Notes in Computer Science, pp. 641-656, Springer, Berlin, Germany, 2003.

[58] A. A. Samsonov and C. R. Johnson, "Noise-adaptive nonlinear diffusion filtering of MR images with spatially varying noise levels," Magnetic Resonance in Medicine, vol. 52, no. 4, pp. 798806, 2004.

[59] K. Krissian and S. Aja-Fernandez, "Noise-driven anisotropic diffusion filtering of MRI," IEEE Transactions on Image Processing, vol. 18, no. 10, pp. 2265-2274, 2009.

[60] R. D. Nowak, "Wavelet-based rician noise removal for magnetic resonance imaging," IEEE Transactions on Image Processing, vol. 8, no. 10, pp. 1408-1419, 1999.

[61] M. E. Alexander, R. Baumgartner, A. R. Summers et al., "A wavelet-based method for improving signal-to-noise ratio and contrast in MR images," Magnetic Resonance Imaging, vol. 18, no. 2, pp. 169-180, 2000. 
[62] P. Bao and L. Zhang, "Noise reduction for magnetic resonance images via adaptive multiscale products thresholding," IEEE Transactions on Medical Imaging, vol. 22, no. 9, pp. 1089-1099, 2003.

[63] C. S. Anand and J. S. Sahambi, "Wavelet domain non-linear filtering for MRI denoising," Magnetic Resonance Imaging, vol. 28, no. 6, pp. 842-861, 2010.

[64] J.-H. Xue, A. Pizurica, W. Philips, E. Kerre, R. Van De Walle, and I. Lemahieu, "An integrated method of adaptive enhancement for unsupervised segmentation of MRI brain images," Pattern Recognition Letters, vol. 24, no. 15, pp. 2549-2560, 2003.

[65] J. C. Dunn, "A fuzzy relative of the ISODATA process and its use in detecting compact well separated clusters," Journal of Cybernetics, vol. 3, no. 3, pp. 32-57, 1974.

[66] J. C. Bezdek, "A convergence theorem for the fuzzy ISODATA clustering algorithms," IEEE Transaction on Pattern Analysis and Machine Intelligence, vol. 2, no. 1, pp. 1-8, 1980.

[67] J. C. Bezdek, R. Ehrlich, and W. Full, "FCM: the fuzzy c-means clustering algorithm," Computers and Geosciences, vol. 10, no. 23, pp. 191-203, 1984.

[68] W. Cai, S. Chen, and D. Zhang, "Fast and robust fuzzy c-means clustering algorithms incorporating local information for image segmentation," Pattern Recognition, vol. 40, no. 3, pp. 825-838, 2007.

[69] L. Szilágyi, S. M. Szilágyi, and B. Benyó, "Efficient inhomogeneity compensation using fuzzy c-means clustering models," Computer Methods and Programs in Biomedicine, vol. 108, no. 1, pp. 80-89, 2012.

[70] IBSR, http://www.cma.mgh.harvard.edu/ibsr/. 

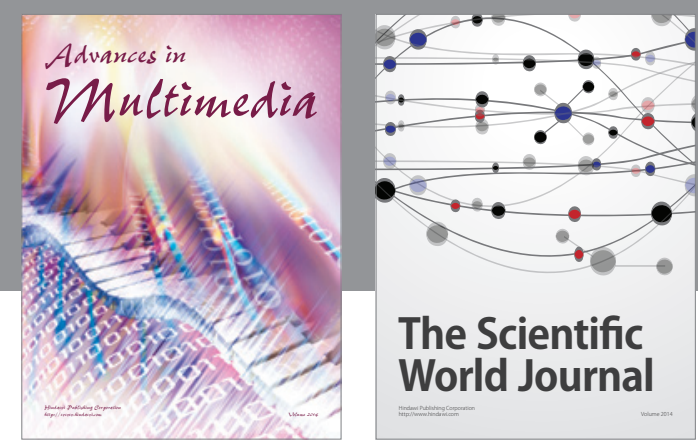

The Scientific World Journal
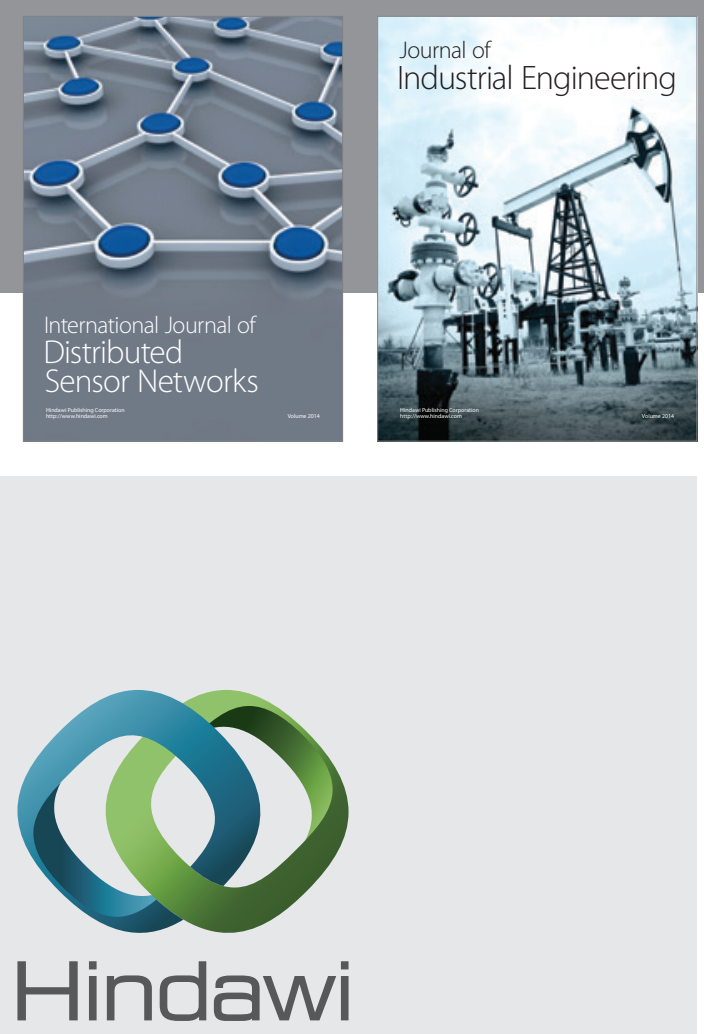

Submit your manuscripts at

http://www.hindawi.com

\section{Computer Networks} and Communications
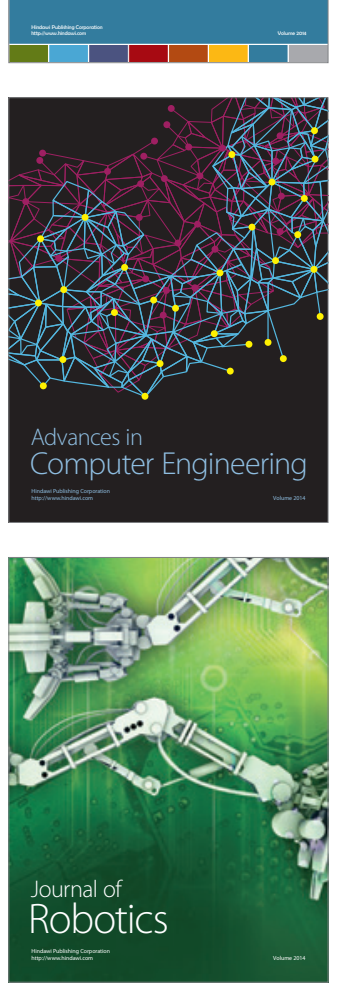
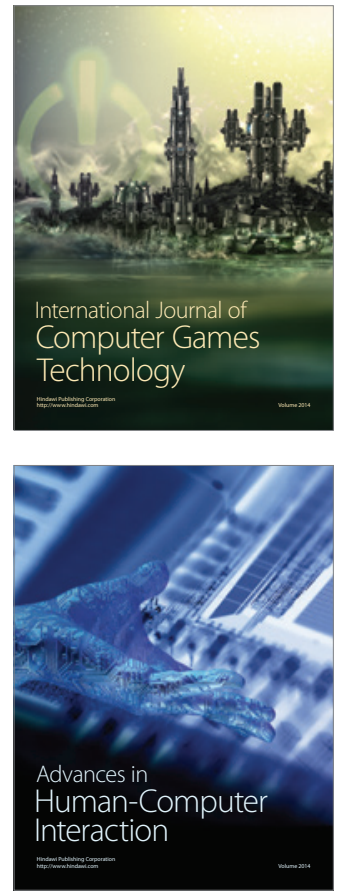
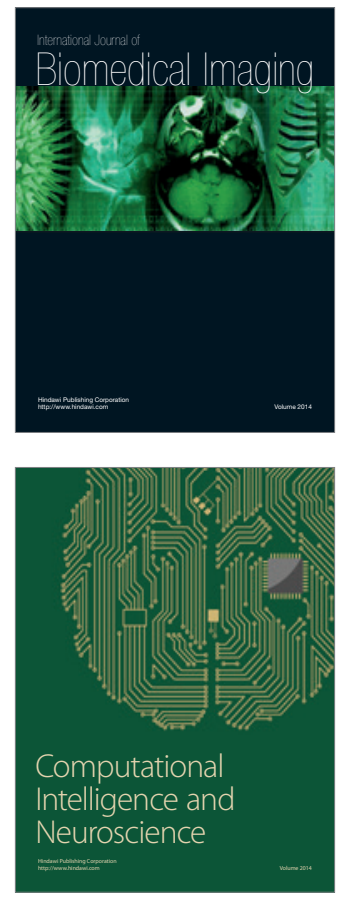
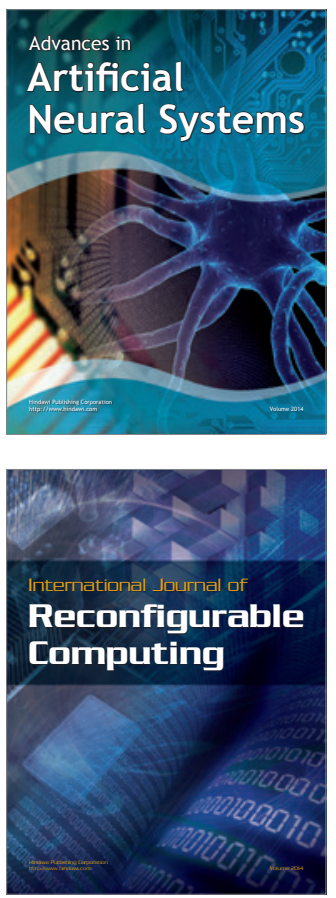
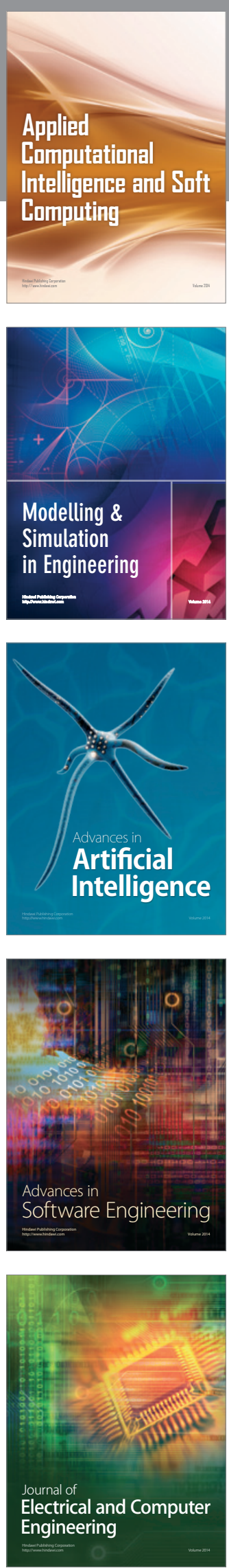